\title{
Avaliação da aceitabilidade de lanche saudável em duas escolas da rede pública
}

\author{
Evaluation of the acceptability of a healthy snack in two public schools \\ Evaluación de la aceptabilidad de una merienda saludable en dos escuelas públicas
}

\author{
Thamires Castro da Silva Pacheco \\ ORCID: https://orcid.org/0000-0002-8943-5734 \\ Universidade Federal do Pará, Brasil \\ E-mail: thamirespacheco.nutri@gmail.com \\ Rosileuda Marques de Souza da Costa \\ ORCID: https://orcid.org/0000-0002-1782-3906 \\ Universidade Federal do Pará, Brasil \\ E-mail: rosileudamarques@gmail.com \\ Eydlane do Rosário Costa Campos \\ ORCID: https://orcid.org/0000-0003-3233-7030 \\ Universidade Federal do Pará, Brasil \\ E-mail: eydlanecampos@gmail.com \\ Xaene Maria Fernandes Duarte Mendonça \\ ORCID: https://orcid.org/0000-0002-0958-276X \\ Universidade Federal do Pará, Brasil \\ E-mail: xaene@ufpa.br \\ Réia Sílvia Lemos da Costa e Silva Gomes \\ ORCID: https://orcid.org/0000-0002-9944-4896 \\ Universidade Federal do Pará, Brasil \\ E-mail:rs.ufpa@gmail.com
}

\begin{abstract}
Resumo
Objetivo: Incentivar a prática de hábitos alimentares saudáveis a partir de metodologias ativas de ensino-aprendizagem com alunos do ensino fundamental de duas escolas municipais de Belém-PA. Métodos: Trata-se de um estudo do tipo transversal, exploratório, descritivo com abordagem quantitativa e qualitativa; conduzido com 82 escolares, de ambos os sexos, na faixa etária de 8 e 14 anos de duas escolas selecionadas (A e B) com o uso da metodologia ativa de oficina de lanche saudável. A Oficina aconteceu nas dependências das escolas participantes, com a elaboração e degustação do lanche saudável: sanduíche natural, suco da horta e uma fruta. Foi aplicado o teste de aceitabilidade da escala hedônica facial e a análise estatística foi planejada no software SAM (Statistical Analysis Model) e os testes de hipótese foram executados no programa BioEstat, versão 5.3. Resultados: $O$ estudo avaliou a aceitabilidade do lanche saudável através da aplicação da escala hedônica facial. A aceitação dos escolares foi de 87,5\% na escola A e de 95,24\% na escola B. Conclusão: Há viabilidade na execução de lanches saudáveis com vegetais cultivados nas hortas escolares municipais e os índices de aceitação expressaram resultados favoráveis à implementação.
\end{abstract}

Palavras-chave: Educação alimentar e nutricional; Degustação; Ensino escolar.

\begin{abstract}
Objective: Encourage the practice of healthy eating habits based on active teaching-learning methodologies with elementary school students from two municipal schools in Belém-PA. Methods: This is a cross-sectional, exploratory, descriptive study with a quantitative and qualitative approach; conducted with 82 students, of both sexes, in age range 8 and 14 years old from two selected schools (A and B) using the active methodology of a healthy snack workshop. The Workshop it happens on the premises of the participating schools, with the preparation and tasting of a healthy snack: natural sandwich, juice from the garden and a fruit. The hedonic facial scale acceptability test was applied and the statistical analysis was planned in the SAM (Statistical Analysis Model) software and the hypothesis tests were performed in the BioEstat program, version 5.3. Results: The study evaluated the acceptability of a healthy snack, through the application of the hedonic facial scale. The acceptance of students was $87.5 \%$ in school A and $95.24 \%$ in school B. Conclusion: There is feasibility in the execution of healthy snacks with vegetables grown in municipal school gardens and the acceptance rates expressed favorable results for the implementation.
\end{abstract}

Keywords: Food and nutrition education; Tasting; School teaching.

\section{Resumen}

Objetivo: Fomentar la práctica de hábitos alimenticios saludables basados en metodologías activas de enseñanzaaprendizaje con estudiantes de primaria de dos escuelas municipales en Belém-PA. Métodos: Este es un estudio transversal, exploratorio, descriptivo con un enfoque cuantitativo y cualitativo; llevado a cabo con 82 escolares, de ambos sexos, de 8 y 14 años de edad de dos escuelas seleccionadas (A y B) utilizando la metodología activa del taller 
de meriendas saludables. El taller tuvo lugar en las instalaciones de las escuelas participantes, con la preparación y degustación de merienda saludable: sándwich natural, jugo de la huerta y una fruta. Se aplicó la prueba de aceptabilidad de la escala facial hedónica y se planificó el análisis estadístico en el software SAM (Statistical Analysis Model) y las pruebas de hipótesis se realizaron en el programa BioEstat, versión 5.3. Resultados: El estudio evaluó la aceptabilidad de una merienda saludable mediante la aplicación de la escala facial hedónica. La aceptación de los escolares fue del 87.5\% en la escuela A y del $95.24 \%$ en la escuela B. Conclusión: Existe factibilidad en la ejecución de botanas saludables con vegetales cultivados en huertos escolares municipales y las tasas de aceptación expresaron resultados favorables para la implementación.

Palabras clave: Educación alimentaria y nutricional; Saboreo; Enseñanza escolar.

\section{Introdução}

A infância é uma importante fase da vida que merece cuidados especializados, pois durante a mesma ocorre período de crescimento, desenvolvimento físico e mental, processos que terão influência durante toda a vida, tendo em vista que se tem a construção de uma condição fundamental para o estabelecimento da saúde do indivíduo pelo comportamento alimentar ou adoção de hábitos de vida saudável. Neste momento que a alimentação adequada e saudável é muitas vezes substituída por refeições inadequadas no âmbito domiciliar com uso de comidas industrializadas ou pela frequência em lanchonetes e fast-foods, tão usuais nos dias de hoje.

O comportamento alimentar infantil pode ser estruturado de diversas formas e as principais influências são advindas do ambiente familiar, da mídia e da escola. No ambiente escolar são valorizadas as relações sociais, como local de sistematização do processo educativo, as práticas pedagógicas dinamizadas facilitam o aprendizado contribuem para a formação de bons hábitos alimentares que assimilados pela educação alimentar perduram por toda uma vida (Soares et al., 2018). Inadequações na alimentação e no consumo de nutrientes na infância comprometem o estado nutricional da criança, pois se ocorrerem carências há aumento da suscetibilidade às diarreias, infecções diversas e comprometimento da maturação corporal e intelectual. Se acontecem excessos nutricionais ele se manifestam através das doenças crônicas não transmissíveis, dentre as quais a obesidade e a diabetes; daí a importância de uma alimentação adequada na infância para o estabelecimento de hábitos alimentares saudáveis que repercutirão na infância, adolescência e vida adulta (Carvalho et al., 2015).

A Educação Alimentar e Nutricional (EAN) é uma área de conhecimento voltada para orientação de hábitos alimentares seguros e saudáveis, no contexto da realização do Direito Humano à Alimentação Adequada e da garantia da Segurança Alimentar e Nutricional. Ela possibilita a utilização de práticas didáticas e recursos educacionais que compartilham com indivíduos e grupos populacionais orientações, de acordo com a faixa etária, sobre as características de cada fase alimentar, as interações com o alimento, seus significados e hábitos culturais, que no seu conjunto compõem o comportamento alimentar daquela população alvo da atividade educativa (Brasil, 2012).

A prática culinária com os escolares, trabalhada como atividade didática de EAN, contribui para geração de conhecimento a respeito de uma alimentação saudável, do valor nutricional dos alimentos e do cuidado com os mesmos, reforçando a importância da alimentação produzida. $\mathrm{O}$ aluno apreendendo essas informações pode adequar o seu hábito alimentar ao usufruir das hortaliças na merenda escolar, pois são partícipes do processo de ensino-aprendizagem, enquanto desenvolvem o cuidado com o alimento, na preparação da alimentação (Santos et al., 2014). De acordo com Rezende \& Negri (2015), a EAN associada às oficinas culinárias para escolares disseminam práticas saudáveis e estimulam o consumo de alimentos mais nutritivos.

O presente trabalho objetiva incentivar a prática de hábitos alimentares saudáveis a partir da adoção de metodologias ativas de ensino-aprendizagem, com foco na educação alimentar, nutricional e sustentável de alunos do ensino fundamental de duas escolas municipais de Belém, capital do Estado do Pará, partindo de métodos básicos de produção, importância do consumo de produtos naturais, educação nutricional e sustentável; ao mesmo tempo que se educa, avaliar o nível de aceitabilidade dos escolares ao lanche proposto, elaborado com hortaliças cultivadas em hortas escolares ou mesmo domiciliares. 


\section{Metodologia}

Estudo transversal, exploratório, descritivo com abordagem quantitativa e qualitativa, autorizado pela Secretaria Municipal de Educação do Município de Belém (SEMEC) e pela direção de duas escolas municipais que possibilitaram o desenvolvimento do projeto de extensão "Cultivando Hortas Escolares como Eixo Gerador de Segurança Alimentar, Nutricional e Sustentabilidade". As Diretoras das escola assinaram o Termo de Consentimento Livre e Esclarecido para a realização do estudo, aprovado sob CAAE no 56171416.1.0000.0018.

A seleção das escolas participantes aconteceu por sorteio entre todas as escolas municipais que possuíam hortas escolares em atividade. As escolas sorteadas foram nomeadas pelas letras A e B e depois feita a seleção de quatro turmas, duas por escola, dentre as turmas do $4^{\circ}$ e $5^{\circ}$ anos de cada uma. A dinâmica proposta como atividade de metodologia ativa de aprendizagem foi a oficina de lanche saudável, potencial geradora de hábitos alimentares saudáveis, realizada durante o mês de junho de 2017 e que trabalhou sobre escolhas de alimentos saudáveis e naturais e consumo de hortaliças cultivadas nas próprias hortas escolares.

A dinâmica da Oficina de Lanche Saudável foi desenvolvida nos refeitórios das escolas participantes e consistiu das seguintes fases: (1) elaboração das preparações propostas pela equipe da pesquisa, sob a assistência curiosa dos escolares das turma sorteadas ; como preparações foram oferecidas: um 'sanduiche natural' à base de patê de frango, pão integral e hortaliças (alface, tomate, pepino, milho, ervilha, cenoura, cebola e cheiro verde), um 'suco da horta' contendo sumo de maracujá, limão, couve; e, banana, como sobremesa; (2) reunião dos escolares no salão do refeitório com apresentação nominal das preparações do lanche e seus componentes, quando informado sobre características alimentares e informações nutricionais; (3) degustação do lanche saudável contendo hortaliças escolares; (4) aplicação do teste de aceitabilidade das preparações consumidas, empregando-se 'escala hedônica facial' utilizada pelo Fundo Nacional de Desenvolvimento da Educação (Brasil, 2009; 2013). A análise estatística foi realizada no software SAM (Statistical Analysis Model) e o programa BioEstat 5.3 adotado para aplicação dos testes estatísticos (Ayres et al., 2007).

\section{Resultados}

Do estudo, participaram 82 escolares, de ambos os sexos, idade variando entre 8 e 14 anos, com predomínio do sexo feminino $(56,1 \%)$ sobre o masculino $(43,9 \%)$. Na avaliação da aceitabilidade do lanche saudável oferecido após as oficinas culinárias a frequência de votação da escala hedônica facial e a aceitação pelos escolares foi de 87,5\% na escola A e de 95,24\% na escola B, que optaram pelos resultados 'Adorei/Gostei'. Não houve diferença estatística significativa entre as escolas, como expresso pelo -valor $=0,1843$.

Nas turmas do $4^{\circ}$ ano (37 escolares participantes) das escolas A e B (Tabela 1), a comparação pelo teste G para amostras independentes, na avaliação da aceitabilidade, observou-se alta aceitabilidade aos lanches: na Escola A o resultado 'Adorei' obteve 78,6 \% e na Escola B o resultado 'Adorei' obteve 78,3 \%; o teste de hipótese resultou no $p$-valor = 0,9764, indicando não haver real diferença entre as notas das escolas. $\mathrm{Na}$ avaliação geral, o teste do Qui-quadrado indicou tendência altamente significante para o resultado 'Adorei' (78,4 \%) e expressivos resultados para a aceitação nas opções 'Gostei/Adorei' (91,9 \%). 
Tabela 1 - Teste de aceitabilidade de lanche escolar saudável em turmas do $4^{\circ}$ ano do Ensino Fundamental de duas escolas públicas do município de Belém/PA (2017).

\begin{tabular}{lcccccc}
\hline $\mathbf{4}^{\mathbf{0}}$ ANO & \multicolumn{2}{c}{ Escola A } & \multicolumn{2}{c}{ Escola B } & \multicolumn{2}{c}{ Geral } \\
Aceitabilidade & $\mathbf{n}$ & $\mathbf{\%}$ & $\mathbf{n}$ & $\mathbf{\%}$ & $\mathbf{N}$ & $\mathbf{\%}$ \\
\hline Detestei & 0 & 0,0 & 0 & 0,0 & 0 & 0,0 \\
Não Gostei & 1 & 7,1 & 0 & 0,0 & 1 & 2,7 \\
Indiferente & 1 & 7,1 & 1 & 4,3 & 2 & 5,4 \\
Gostei & 1 & 7,1 & 4 & 17,4 & 5 & 13,5 \\
Adorei & 11 & 78,6 & 18 & 78,3 & 29 & 78,4 \\
Total & 14 & 100,0 & 23 & 100,0 & 37 & 100,0 \\
\hline p-valor $=0.9764$, Teste G, diferença não significante & & & \\
p-valor $<0.0001 *$, Qui-quadrado de tendência, para Adorei $78,4 \%$ & \\
\hline
\end{tabular}

Fonte: Autores (Pacheco et al., 2017).

$\mathrm{Na}$ avaliação da aceitabilidade nas turmas do $5^{\circ}$ ano (45 escolares), comparando-se as escolas A e B (Tabela 2), o teste G expressou alta aceitabilidade, pois o resultado ‘Adorei’ na Escola A expressou 76,9 \% e na Escola B 78,9 \%; o teste de hipótese $(\mathrm{p}$-valor $=0.9861)$ aponta que não houve real diferença entre as escolas. Na avaliação geral o teste do Qui-quadrado foi altamente significante $\left(\mathrm{p}<0.0001^{*}\right)$, tendente ao resultado 'Adorei' $(77,8 \%)$ e ampla aceitação geral do lanche, expresso por resultados 'Gostei/Adorei' (90,1\%).

Tabela 2 - Teste de aceitabilidade de lanche escolar saudável em turmas do $5^{\circ}$ ano do Ensino Fundamental de duas escolas públicas do município de Belém/PA (2017).

\begin{tabular}{lcccccc}
\hline \multicolumn{1}{c}{$\mathbf{5}^{\mathbf{2}}$ ANO } & \multicolumn{2}{c}{ Escola A } & \multicolumn{2}{c}{ Escola B } & \multicolumn{2}{c}{ Geral } \\
Aceitabilidade & $\mathbf{n}$ & $\mathbf{\%}$ & $\mathbf{n}$ & $\mathbf{\%}$ & $\mathbf{N}$ & $\mathbf{\%}$ \\
\hline Detestei & 1 & 3,8 & 0 & 0,0 & 1 & 2,2 \\
Não Gostei & 0 & 0,0 & 0 & 0,0 & 0 & 0,0 \\
Indiferente & 2 & 7,7 & 1 & 5,3 & 3 & 6,7 \\
Gostei & 3 & 11,5 & 3 & 15,8 & 6 & 13,3 \\
Adorei & 20 & 76,9 & 15 & 78,9 & 35 & 77,8 \\
Total & 26 & 100,0 & 19 & 100,0 & 45 & 100.0 \\
\hline p-valor $=0.9861$, Teste G, diferença não significante & & & \\
p-valor < $0.0001 *$, Qui-quadrado de tendência, para Adorei $77,8 \%$ & & \\
\hline
\end{tabular}

Fonte: Autores (Pacheco et al., 2017).

\section{Discussão}

A fase escolar da criança, na educação básica, começa em torno dos 7 anos, um período importante no desenvolvimento da criança, uma vez que muda seu relacionamento com a comida e com as pessoas e passa a ter maior intensidade em suas relações sociais (Benetti, 2013). Mídias como a televisão, computador, tablet e celular convertem-se em entretenimento para crianças e podem interferir no comportamento alimentar e, pelo fato de pais trabalharem diariamente, as crianças ficam expostas às situações de acomodação a essa cotidianidade e mais cedo consomem uma alimentação desequilibrada (Ceccatto et al., 2018).

O Programa Nacional de Alimentação Escolar (PNAE) é uma política pública de alimentação muito antiga e que ao longo do tempo oportunizou espaço de ação destacado ao nutricionista, desde a obrigatoriedade da responsabilidade técnica do programa nas entidades executoras, desde 2006 (Souza et al., 2017). Nesse programa as atribuições do nutricionista não são 
somente as de planejar e elaborar cardápios adequados e nutricionalmente balanceados às demandas nutricionais dos escolares, mas devem observar o perfil epidemiológico da população atendida, respeitar a cultura alimentar local, garantir a segurança alimentar e nutricional (Brasil, 2009; 2020), implementar projetos de educação alimentar e nutricional para a comunidade escolar, inclusive no desenvolvimento da consciência social, ecológica e ambiental (Brasil, 2009; CFN, 2018), como estímulo ao consumo de produtos locais, o que valoriza a cultura alimentar e o desenvolvimento sustentável.

Nessa faixa etária as crianças estão crescendo e desenvolvendo ossos, dentes, músculos, sangue e precisam de alimentos compatíveis com as proporções do seu tamanho, mais nutritivos do que aqueles de adultos, contendo nutrientes como proteínas, minerais como o ferro, cálcio, zinco e as vitaminas D, C e outros elementos imprescindíveis ao desenvolvimento físico, motor e cognitivo (Mahan et al., 2013). Ações dinâmicas como as oficinas de culinária e gastronomia propondo refeições mais saudáveis nessa fase infantil, utilizando alimentos adequados e ricos em nutrientes essenciais, por envolver as crianças no processo do aprender fazer-fazendo são significantes para estimular boas escolhas, preferências alimentares e contribuir para o desenvolvimento integral de corpo e mente.

Como esses escolares passam a ser bombardeados com influências negativas relacionadas à alimentação, com consequente aumento do consumo de alimentos ricos em gorduras, açucares, sódio e pobres em fibras e nutrientes, as atividades de ensino-aprendizagem com hortas envolvem os escolares de maneira lúdica na aprendizagem dos conhecimentos sobre os diferentes tipos de alimentos e comportamentos alimentares saudáveis; bem como, permitem problematizar e compreendam que a falta, o excesso ou as escolhas inadequadas impactam na saúde de cada um deles. As estratégias de EAN são relevantes, primordialmente, na infância e na adolescência, daí a importância do ambiente escolar como um espaço gerador de hábitos alimentares, como provado nos estudos semelhantes ao proposto nas duas escolas trabalhadas no município de Belém, cujos resultados revelaram boa aceitação do lanche saudável, como a seguir se registra.

Em uma escola de Curitiba, Estado do Paraná, Jesus et al. (2016) trabalharam estratégias de EAN a partir da preparação de um cupcake de vegetais (cenoura, brócolis, couve-flor, espinafre) e utilizaram a escala hedônica facial para o teste de aceitabilidade. A expressão dos resultados 'gostei + adorei' foi de 90,40 \%, um padrão muito positivo de aceitação do lanche proposto. Cain et al. (2020) realizaram seus estudos em 10 escolas públicas de ensino básico na cidade de Guarapuava, Estado do Paraná, em amostra de 1.010 escolares de ambos os sexos. Esses pesquisadores organizaram oficinas culinárias com alimentos nutritivos menos aceitos pelo grupo; realizaram todo um trabalho de EAN, de preparação culinária, oferta dos lanches para degustação e aplicação de escala hedônica facial de sete e de cinco pontos e obtiveram resultados com índices de aceitabilidade acima de $70 \%$.

Basaglia et al. (2015) aplicaram o teste de aceitabilidade em 12 escolas estaduais do município de Amparo, Estado de São Paulo, e observaram que a resposta na escala hedônica facial é mais favorável $(83,72 \%)$ do que para a escala hedônica verbal (64,55\%), pois a expressão 'Indiferente', nesta última, foi a segunda mais escolhida (21,51\%) e constataram que as crianças aceitaram bem produtos que diferem de seus hábitos alimentares, pois não rejeitaram alimentos ricos em fibras, como o pão integral e as hortaliças, oferecidos como lanche. No município de Dois Irmãos, Estado do Rio Grande do Sul, foram feitas ações de EAN sustentável em escolas com hortas escolares ativas e que expressaram dados positivos na questão do consumo alimentar mais saudável e na vigilância do peso dos escolares entre 8 e 10 anos de idade, em estudo longitudinal que durou sete anos e verificou o aumento na proporção de escolares com peso adequado, de 76,4 \% em 2003 para 81,3 \% em 2010 (Triches, 2015).

O Fundo Nacional de Desenvolvimento da Educação (FNDE) faz repasses financeiros ao Programa Nacional de Alimentação Escolar que busca atender às necessidades nutricionais dos alunos durante sua permanência em sala de aula. Como gestor do programa, o FNDE estabeleceu como padrão para os índices pesquisados nos testes de aceitabilidade com escala hedônica facial/escrita que a soma dos resultados da escala para as expressões '4- gostei e 5-adorei' deve atingir o mínimo de 
85\% para ser considerada de boa aceitação (Brasil, 2013). No programa de alimentação escolar cabe ao nutricionista incentivar o consumo de hortaliças em diversas formas de preparações culinárias, principalmente, naquelas escolas que têm em seus planos pedagógicos programas ativos de hortas escolares.

Estudos realizados com hortas escolares indicam que estas podem ser usadas como ferramentas estratégicas para aumentar o acesso à educação em saúde e complementar programas de educação nutricional e sustentável nas escolas (Turner et al, 2017); além do que, as hortas ativas contribuem para que a criança estabeleça uma relação diferente com os alimentos através da curiosidade sobre a cadeia alimentar e a origem dos alimentos (Coelho \& Bógus, 2016). Para Carlsson et al. (2016), o aluno ao comer alimentos por ele cultivados, mas nunca provados, despertam um sentimento de orgulho, de propriedade e vontade de provar e comer algo novo. Esse pertencimento pode ser potencializado com oficinas culinárias que utilizem os produtos cultivados na horta.

Muzaffar et al. (2018) demonstraram haver uma relação otimista nas experiências de intervenções culinárias com escolares, pois resultaram na melhoria do consumo alimentar e mudanças positivas nas avaliações antropométricas; por isso, sugeriram que o programa de merenda escolar seja objeto de pesquisas com metodologias padronizadas para avaliar os efeitos a longo prazo das intervenções culinárias na saúde dos escolares. Rezende \& Negri (2015) consideram que a EAN estimula a reeducação alimentar com o consumo de alimentos mais nutritivos e proporciona aos alunos conhecimentos em alimentação e nutrição, com influências sobre as preferências dos escolares. Para Silva et al. (2018), a presença do nutricionista está associada à EAN; contudo, esse profissional e estas ações são executadas com baixa frequência pelas escolas. Rabelo et al. (2020) demonstraram que projetos de extensão universitária possibilitam compartilhar os conhecimentos da academia e motivar escolares, educadores, gestão escolar e comunidade a se apropriarem de conhecimentos que conduzirão à segurança alimentar e nutricional dos envolvidos.

Nos trabalhos referidos e no presente estudo observou-se que a estratégia da educação alimentar é uma forma eficaz e positiva de promoção da saúde e que as atividades didáticas no formato de oficinas de práticas culinárias, experimentando a preparação de refeições e lanches com qualidade nutricional e segurança alimentar. Nessas atividades as crianças são educadas, estimuladas a conhecer, manusear e relacionar-se de forma saudável com o alimento; assim, obtendo grande adesão às ações praticadas e cujos índices dos testes de aceitabilidade das preparações atingiram 91,4 \%, numa expressiva aceitação do lanche proposto pela equipe de pesquisa.

\section{Considerações Finais}

Artigos utilizando a Educação Alimentar e Nutricional são numericamente relevantes, mas a associação da EAN com testes de aceitabilidade da alimentação escolar, após intervenções dinamizadas pela confecção de lanches com produtos de hortas escolares ainda são escassos no Brasil, o que possibilita a esse estudo uma diferenciação ao valorizar as hortas escolares como eixo gerador de segurança alimentar, nutricional e sustentabilidade local.

A aceitabilidade do lanche pelos escolares foi alta e a equipe acredita que os escolares por terem acompanhado e auxiliado em alguma das etapas da oficina, seja na colheita das hortaliças, na preparação dos lanches, arranjo do espaço, interrogações curiosas da natural curiosidade da infância, ouvindo explicações pari-passu às etapas de confecção do lanche até a apresentação como EAN para o grupo de escolares e docentes sobre a importância de cada alimento e do lanche saudável, comentando as propriedades nutricionais de cada preparação; a visão da expressão de satisfação dos escolares após a degustação e a empolgação em participar do teste de aceitabilidade.

Convém que nutricionistas, merendeiras, professores, diretores e pais de alunos trabalhem em conjunto para viabilizar atividades planejadas desde a elaboração do projeto pedagógico das escolas da educação básica e que estes tenham opção pelas oficinas pedagógico-culinárias como ferramentas de envolvimento estudantil e fácil execução. Esse tipo de educação para uma 
alimentação saudável com legumes, verduras e, quem sabe, até frutas provindas de hortas e pomares das escolas pode mudar hábitos alimentares e o estado de saúde dos escolares, resguardando-os de doenças de desenvolvimento insidioso, que poderão surgir na adolescência ou idade adulta.

\section{Agradecimentos}

À Pró-Reitoria de Extensão da Universidade Federal do Pará por possibilitar a realização deste estudo através da concessão de bolsas captadas no Edital Proex nº 6/2017 - Eixo Transversal. Agradecemos às Diretoras das Escolas Municipais, coordenadoras pedagógicas e merendeiras, pela acolhida. Às nutricionistas e voluntárias do projeto por auxiliarem na aplicação dos testes e na análise dos dados coletados.

\section{Referências}

Ayres, M., Ayres Jr, M., Ayres, D. \& Santos, A. A. S. (2007). BioEstat: Aplicações Estatísticas nas áreas das Ciências Biológicas e Médicas. $5^{a}$ ed. Brasília/DF: Mamirauá, 2007.

Benetti, G.B (2013). Curso Didático de Nutrição, Yendis.

Basaglia, P., Marques, A. S. \& Benatti, L. (2015). Aceitação da merenda escolar entre alunos da Rede Estadual de Ensino, da Cidade de Amparo-SP. Saúde em Foco, 07:126-138. https://portal.unisepe.com.br/unifia/wp-content/uploads/sites/10001/2018/06/16aceitacao_merenda.pdf

Brasil. Presidência da República (2020). Lei $n^{o} 13.987$, de 7 de abril de 2020. Altera a Lei $n^{\circ} 11.947$, de 16 de junho de 2009 , para autorizar, em caráter excepcional, durante o período de suspensão das aulas em razão de situação de emergência ou calamidade pública, a distribuição de gêneros alimentícios adquiridos com recursos do Programa Nacional de Alimentação Escolar (Pnae) aos pais ou responsáveis dos estudantes das escolas públicas de educação básica.

Brasil. Fundo Nacional de Desenvolvimento da Educação (2013). Resolução $n^{\circ}$ 26, de 17 de junho de 2013. Dispõe sobre o atendimento da alimentação escolar aos alunos da educação básica no âmbito do Programa Nacional de Alimentação Escolar - PNAE.

Brasil. Ministério do Desenvolvimento Social e Combate à Fome (2012). Marco de Referência de Educação Alimentar e Nutricional para as Políticas Públicas. Brasília/DF: MDS. https://www.cfn.org.br/wp-content/uploads/2017/03/marco_EAN.pdf

Brasil. Presidência da República (2009). Lei $n^{o} 11.947$, de 16 de junho de 2009. Dispõe sobre o atendimento da alimentação escolar e do Programa Dinheiro Direto na Escola aos alunos da educação básica, altera as Leis no 10.880 , de 9 de junho de 2004, $\mathrm{n}^{\circ} 11.273$, de 6 de fevereiro de 2006 , n ${ }^{\circ} 11.507$, de 20 de julho de 2007, revoga dispositivos da Medida Provisória nº 2.178-36, de 24 de agosto de 2001, e a Lei nº 8.913, de 12 de julho de 1994, e dá outras providências.

Cain, J. P., Silva, V. C., Franco, B. C., Luz, L. A. P., Santos, E. F. \& Novello, D. (2020). Oficinas de culinária melhoram a aceitabilidade de alimentos entre crianças de idade escolar. Res Soc Dev, 9(4):e20942952, 2020. http://dx.doi.org/10.33448/rsd-v9i4.2952

Carlsson, L., William, P. L., Hayes-Conroy, J. S., Lordly, D. \& Callaghan, E. (2016). School gardens: cultivating Food Security in Nova Scotia Public Schools? Can J Diet Pract Res, 77:119-124. https://doi.org/10.3148/cjdpr-2015-051

Carvalho, C. A., Fonsêca, P. C. A., Priore, S. E., Franceschini, S .C. C. \& Novaes, J. F. (2015). Consumo alimentar e adequação nutricional em crianças brasileiras: revisão sistemática. Rev Paul Pediatr, 33(2):211-221. https://doi.org/10.1016/j.rpped.2015.03.002

Cecatto, D., Spinelli, R. B., Zanardo, V. P. S. \& Ribeiro, L. A. (2018). A influência da mídia no consumo alimentar infantil: uma revisão da literatura. Perspectiva, Erechim, 42(157):141-149. https://www.uricer.edu.br/site/pdfs/perspectiva/157_700.pdf

CFN. Conselho Federal de Nutricionista (2018). Resolução $n^{\circ} 600$, de 25 de fevereiro de 2018. Dispõe sobre a definição das áreas de atuação do nutricionista e suas atribuições, indica parâmetros numéricos mínimos de referência, por área de atuação, para a efetividade dos serviços prestados à sociedade e dá outras providências.

Coelho, D. E. P. \& Bógus, C. M. (2016). Vivências de plantar e comer: a horta escolar como prática educativa, sob a perspectiva dos educadores. Saúde Soc. São Paulo, 25(3):761-771. http://10.1590/S0104-12902016149487

Jesus, A. M. F., Barros, A. C. A. \& Mattanna, P. (2016). Aceitabilidade de cupcake de vegetais por crianças em uma escola municipal de Curitiba/PR. Biociências, Biotecnologia e Saúde, Curitiba, 7(16):38-49. https://interin.utp.br/index.php/GR1/article/view/1587

Mahan, K. L., Escott-Stump, S. \& Raymond, J.L. (2013). Krause Alimentos, Nutrição e Dietoterapia. Elsevier.

Muzaffar, H., Metcalfe, J. J. \& Fiese, B. (2018). Narrative review of culinary interventions with children in schools to promote healthy eating: directions for future research and practice. Curr Dev Nutr, 26,2(6):nzy016. http://10.1093/cdn/nzy016.

Rabelo, C. A. F, Mendes, A. E. P., Souza, F. E. B., Lima, D. S., Amaral, R. Q. G., Bezerra, J. A. B. \& Sousa, P. H. M. (2020) Promoção da segurança alimentar e nutricional em escolas de ensino médio em tempo integral: relato de experiência extensionista. Res Soc Dev, Itajubá, 9(12), e13691210835 (CC BY 4.0) ISSN 2525-3409. http://dx.doi.org/10.33448/rsd-v9i12.10835.

Rezende, M. F. \& Negri, S. T. (2015) Educação Alimentar e Nutricional associada a oficinas culinárias com alunos em uma escola pública. Extensio Florianópolis, 12(20):21-35. https://doi.org/10.5007/1807-0221.2015v12n20p21 
Research, Society and Development, v. 10, n. 9, e21010917852, 2021

(CC BY 4.0) | ISSN 2525-3409 | DOI: http://dx.doi.org/10.33448/rsd-v10i9.17852

Santos, M. J. D., Azevedo, T. A. O., Freire, J. L. O., Arnaud, D. K. L. \& Reis, F. L. A. M. (2014). Horta escolar agroecológica: incentivadora da aprendizagem e de mudanças de hábitos alimentares no ensino fundamental. Holos, 30(4):278-290. https://doi.org/10.15628/holos.2014.1705

Silva, S. U., Monego, E. T., Sousa, L. M. \& Almeida, G. M (2018). As ações de educação alimentar e nutricional e o nutricionista no âmbito do Programa Nacional de Alimentação Escolar. Cien Saude Colet, 23(8):2671-2681. https://10.1590/1413-81232018238.19642016

Soares P., Martinelli, S. S., Fabri, R. K., Veiros, M., Davó-Blanes, M. C. \& Cavalli, S. B. (2018). Programa Nacional de Alimentação Escolar como promotor de sistemas alimentares locais, saudáveis e sustentáveis: uma avaliação da execução financeira. Cienc Saude Colet, 23(12):4189-4197. 10.1590/1413812320182312.25582016

Souza, A. A., Bezerra, O. M. P. A., Bonomo, E. \& Silva, C.A.M. (2017) Atuação de nutricionistas responsáveis técnicos pela alimentação escolar de municípios de Minas Gerais e Espírito Santo. Cien Saude Colet, 22(2):593-606. https://doi.org/10.1590/1413-81232017222.12272016

Triches, R. M. (2015). Promoção do consumo alimentar sustentável no contexto da alimentação escolar. Trab Educ Saude, 13(3):757-771. https://doi.org/10.1590/1981-7746-sip00061

Turner, L., Leider, J., Piekarz, E., Schermbeck, R. M., Merlo, C., Brener, N. \& Chriqui J. F. (2017). Facilitating fresh: state laws supporting school gardens are associated with use of garden-grown produce in School Nutrition Services Programs. J Nutr Educ Behav (2018), 49(6):481-489.e1. 10.1016/j.jneb.2017.03.008 